\title{
Tomato, a Potential Yin Food, Protects against Stroke
}

\author{
Jintanaporn Wattanathorn ${ }^{1,2,3 *}$, Wipawee Thukham-mee ${ }^{1,2}$, Supaporn Muchimapura ${ }^{1,2,3}$, \\ Terdthai Tong-Un ${ }^{1,2}$, Panakaporn Wannanon ${ }^{1,2}$ \\ ${ }^{1}$ Department of Physiology, Faculty of Medicine, Khon Kaen University, Khon Kaen, Thailand \\ ${ }^{2}$ Integrative Complimentary and Alternative Medicine Research Group, Khon Kaen University, Khon Kaen, Thailand \\ ${ }^{3}$ Stroke Research Group, Khon Kaen University, Khon Kaen, Thailand \\ Email: jintanapornw@yahoo.com
}

Received June 4, 2012; revised July 3, 2012; accepted July 15, 2012

\begin{abstract}
Backgroud: Ischemic stroke is one of the challenge strategies nowadays due to the limitation of therapeutic efficacy. Yin deficiency and oxidative stress have been implicated in the mechanism of brain damage induced by cerebral ischemia. Therefore, we aimed to investigate whether tomato, a "Yang food" possessing antioxidant, may ameliorate brain damage in focal cerebral ischemia induced by right middle cerebral artery occlusion. Methods: Male rats were orally given tomato pomace (contained total phenolic compound $413.07 \pm 34.45 \mathrm{mg}$ of gallic acid equivalent $/ \mathrm{mg}$ ) at doses of 2 , 19 and $50 \mathrm{mg} / \mathrm{kg}$ at a period of 2 weeks before and 3 weeks after the occlusion of right middle cerebral artery. Then, they were determined the brain ischemic volume and neurological score. Moreover, the markers of oxidative stress including malondialdehyde level and the activities of superoxide dismutase, catalase and glutathione peroxidase in hippocampus, striatum and cerebral cortex were also determined. Result: The results showed that tomato pomace could mitigate brain damage and neurological impairment. The neuroprotective mechanism might occur partly via the decreased oxidative stress. Conclusion: Tomato may provide health benefit for those who are risk for stroke. However, further researches are required.
\end{abstract}

Keywords: Tomato; Lycopersicon escalentum; Focal Cerebral Ischemia; Brain Damage

\section{Background}

Stroke is a leading cause of death and functional disability in adult [1]. The most commonly found ischemic stroke is focal cerebral ischemia. Previous study showed that approximate the middle cerebral artery occlusion occurred in $10 \%-15 \%$ of stroke patient. The cerebral ischemia has been characterized by a rapid onset of neurological injury due to interruption of blood flow to the brain [2]. Accumulating lines of evidence have suggested that free radicals play a significant role in the development of brain injury following cerebral ischemia [3]. However, stroke is divided into 2 main types according to Traditional Chinese Medicine. Excess stroke usually occurs during an activity and involves bleeding and high blood pressure or blood vessels disorders or brain tumor or trauma whereas deficiency stroke usually occurs been shown that once a stroke occurs, no pharmacological treatment has been available to prevent subsequent during quiet time and involves the blockage with blood stasis, embolisms or fat and phlegm stasis or trauma. It has brain damage, although some experimental drugs with potential promise are emerging [4]. Due to the limitation

\footnotetext{
"Corresponding author.
}

of stroke therapy, the development of novel protective strategy is required. Numerous approaches have been performed including complimentary alternative medicine.

Food has been long term used as medicine in Traditional Chinese Medicine (TCM) for a long time. Foods in Chinese Medicine are categorized as hot, cold or neutral foods depending on their effects on the body. "Yin foods" are cooling foods and are used to offset too much while "Yang foods" are warming foods which exert the opposite effect as mentioned earlier and neutral foods provide a balance between Yin and Yang.

According to TCM, foods which supply concentrated nutrition to maintain or improve tissue integrity, and encourage increased fluid and moisture levels are recommended for Yin deficiency disorders. Since ischemic stroke appears to be Yin deficiency and hyperactivity of yang, the beneficial effect of "Yang food" is considered [5].

Tomato or Lycopersicon escalentum Mill, a plant in a family of Solanaceae, is regarded as "Yin or cooling food". In Chinese Medicine, it is believed that tomato have the effectiveness of detoxification, reducing blood pressure, preventing from cancer and aging. It is also rich 
in substances providing health benefits such as lycopene, phenolic compound, Vitamin A and Vitamin C [6,7]. In addition, it is also served as a major source of antioxidant [8]. Thus, this raised the hypothesis that the tomato, the "Yang food", should have the capability to protect against brain damage induced by focal cerebral ischemia. To the best of our knowledge, no scientific evidence is available until now. Therefore, this study was carried out to determine the protective effect of tomato pomace powder against focal cerebral ischemia induced by middle cerebral artery occlusion.

\section{Methods}

\subsection{Preparation of Dried Tomato Pomace Powder}

Wet tomato pomace powder consisting of tomato skins, pulp and crushed seeds that remain after the processing of tomato for juice was dried with oven at 50 degree Celsius for 2 hours. Then, it was grinded as powder and determined total phenolic compound and used as marker for quality control. The dried tomato pomace powder at the percent yield of 12.75 was kept in airtight container at room temperature until use. The administration of dried tomato pomace powder was performed using propylene glycol as vehicle.

\subsection{Determination of Total Phenolic Content}

The total phenolic content of extracts was determined using to the Folin-Ciocalteu method [9]. The extracts were oxidized with Folin-Ciocalteu reagent, and the reaction was neutralized with sodium carbonate. The absorbance of the resulting blue color was measured at $760 \mathrm{~nm}$ after $60 \mathrm{~min}$. Using gallic acid (GA) as standard total phenolic content (standard curve was prepared using concentrations of $2.5-50 \mathrm{mg} / \mathrm{ml}$ ) was expressed as $\mathrm{mg}$ GA equivalent/mg of extract. Data reported of three replications.

\subsection{Animals}

Male Wistar rats weighing 300 - 350 gm were obtained from National Laboratory Animal Center, Salaya, Nakorn Pathom, and were housed in group of 5 per cage in standard metal cages at $22^{\circ} \mathrm{C} \pm 2{ }^{\circ} \mathrm{C}$ on $12: 12 \mathrm{~h}$ light-dark cycle. All animals were given access to food and water ad libitum. The experiments were performed to minimize animal suffering in accordance with the internationally accepted principles for laboratory use and care of European Community (EEC directive of 1986; 86/609/EEC). The experimental protocols were approved by the Institutional Animal Care and Use Committee.

\subsection{Experimental Protocol}

In order to induce focal cerebral ischemia, all rats in this study were occluded the right middle cerebral artery oc- clusion (MCAO). Rats were randomly divided into various groups as described following: 1) Vehicle + MCAO; 2) Vitamin $\mathrm{C}+\mathrm{MCAO}$ (Vitamin $\mathrm{C}+\mathrm{MCAO}$ served as positive control); 3)-5) Tomato $+\mathrm{MCAO}(\mathrm{T} 2, \mathrm{~T} 10$ and T50 + MCAO). The animals in group 2) were treated with Vitamin C (250 mg/kg BW), a well known antioxidant which previously reported to exert the neuroprotective effect. The animals in group 3)-5) were treated with tomato pomace powder at doses of 2,10 and $50 \mathrm{mg} / \mathrm{kg}$ $\mathrm{BW}$ respectively. The dosage range of tomato pomace powder used in this study was selected based on the previous data about the effect of tomato on the central nervous system and Vitamin $\mathrm{C}$ was used as positive control because it has been previously demonstrated to exert the neuroprotective effect. All administered substances used in this study were freshly prepared and used propylene glycol as vehicle.

\subsection{Focal Cerebral Ischemia Induction}

All rats were fasted for $12 \mathrm{hr}$ but they were allowed free access to water before surgery. Anesthesia was induced with intraperitoneal injection of thiopental sodium at dose of $50 \mathrm{mg} / \mathrm{kg}$ BW. Focal cerebral ischemia was induced by modified previous method described by Longa et al. Briefly, the right common carotid artery and the right external carotid artery were exposed through a ventral midline neck incision and were ligated proximally. A silicone coated nylon monofilament (4-0) suture (USS $\mathrm{DG}^{\mathrm{TM}}$ sutures; Tyco Healthcare group LP, Connecticut, USA) with its tip rounded by heating near a flame was inserted through an arteriectomy in the common carotid artery just below the carotid bifurcation, and then advanced into the internal carotid artery approximately 17 to $18 \mathrm{~mm}$ distal to the carotid bifurcation until a mild resistance was felt. Occlusion of the origins of the anterior cerebral artery, the middle cerebral artery, and the posterior communicating artery was thereby achieved. Then, the wound was sutured, the rats were returned to their cages with free access to food and water. The incision sites were infiltrated with $10 \%$ Povidone-Iodine Solution for anti-septic postoperative care.

\subsection{Determination of Infarct Volume}

All animals were killed 24 hours after occlusion, and the brain was removed and sectioned at $2-\mathrm{mm}$ thick. Sections were immersed in $2 \%$ TTC (2,3,5-triphenyl tetrazolium chloride) for 30 minutes at $37^{\circ} \mathrm{C}$. Images of stained sections were digitized, and infarction volumes were determined using an indirect method.

\subsection{Determination of Antioxidant Enzymes Activities}

In order to determine the activities of antioxidant enzymes including superoxide dismutase (SOD), catalase 
(CAT) and glutathione peroxidase (GSH-Px), the ischemic brains tissues were weighed and homogenized with a buffer consisting of $10 \mathrm{mM}$ sucrose, $10 \mathrm{mM}$ Tris $\mathrm{HCl}$, and $0.1 \mathrm{mM}$ EDTA (pH 7.4), and then centrifuged at $3000 \mathrm{~g}$ for $15 \mathrm{~min}$ at $4^{\circ} \mathrm{C}$. The supernatant was used for bioassays. The activity of SOD was determined using a xanthine/xanthine oxidase system for production of superoxide radical and subsequent measurement of cytochrome $c$ as a scavenger of the radicals. Optical density was determined using spectrometer (UV-1601, Shimadzu) at $550 \mathrm{~nm}$. One unit of enzyme activity was defined as the quantity of SOD required to inhibit the rate of reduction of cytochrome $c$ by $50 \%$. SOD activity is presented as units per milligram of protein (U/mg protein). Catalase activity in the supernatant was measured by recording the rate of decrease in $\mathrm{H}_{2} \mathrm{O}_{2}$ absorbance at $240 \mathrm{~nm}$. The activity of catalase was expressed as $\mu \mathrm{mol} \mathrm{H}_{2} \mathrm{O}_{2} / \mathrm{min} / \mathrm{mg}$ protein. GSH-Px was determined using t-butylhydroperoxide as a substrate. The optical density was spectrophotometrically recorded at $340 \mathrm{~nm}$. One unit of the enzyme was defined as micromoles ( $\mu \mathrm{mol})$ of reduced nicotinamide adenine dinucleotide phosphate (NADPH) oxidized per minute. The GSH-Px activity was expressed as U/mg protein.

\subsection{Determination of the Malondialdehyde Level}

Various brain brains of the rats including cerebral cortex, striatum and hippocampus were isolated and prepared as brain homogenate as mentioned earlier. Then, the level of lipid peroxidation in the brain homogenate was indirectly estimated by determining the accumulation of thiobarbituric acid reactive substances (TBARS).

\subsection{Statistical Analysis}

Data were presented as mean \pm standard error of mean (S.E.M.). The analysis was performed using one-way analysis of variance (ANOVA), followed by Dunnett's test. All statistical results were considered significant if p-value $<0.05$.

\section{Results}

\subsection{Total Phenolic Compound of Tomato Pomace Powder}

The amount of total phenol was determined with the Folin-Ciocalteu reagent. Gallic acid was used as a standard compound and the total phenols were expressed as mg of Gallic acid equivalent. Phenolic compounds had been regarded as antioxidant agents which acted as free radical terminators [10]. It was found that the dried tomato pomace powder used in this study contained total phenolic compound at the concentration of $413.07 \pm 34.45 \mathrm{mg}$ of gallic acid equivalent $/ \mathrm{mg}$.

\subsection{Neuroprotective Effect of Tomato Pomace Powder}

Figure 1 showed that before the induction of cerebral ischemia, no significant changes of the neurological score. All groups still showed grade 5 of the neurological score. After the induction of neurological score, all treated groups showed the reduction of neurological score. At 7 days after the occlusion of right middle cerebral artery, rats which subjected to Vitamin $\mathrm{C}$ treated groups significantly enhanced the neurological score $(\mathrm{p}$-value $<0.01)$

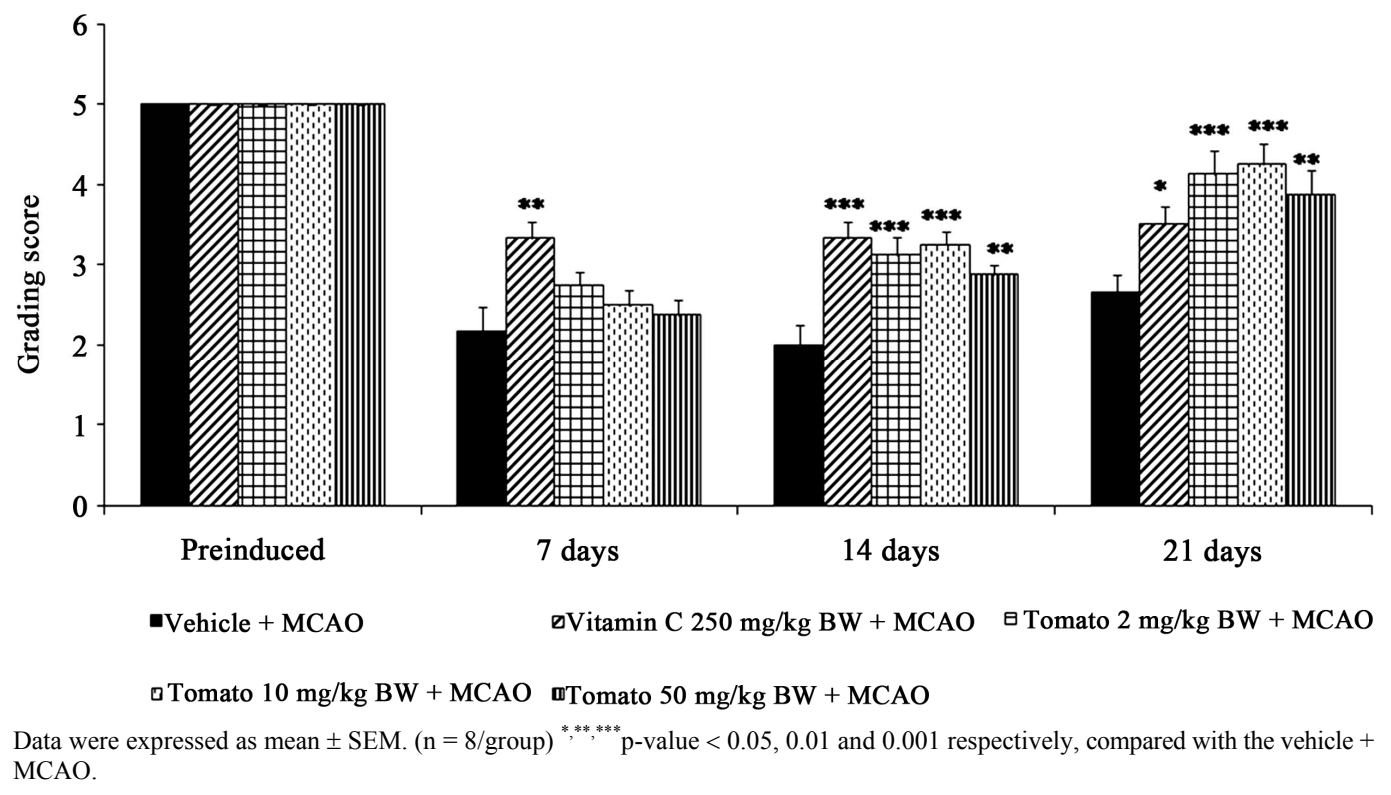

Figure 1. The neurological score of rats treated with various doses of tomato pomace powder at doses of 2,10 and $50 \mathrm{mg} / \mathrm{kg}$ BW at 14 days before and 21 days after the occlusion of right middle cerebral artery (MCAO). 
compared to vehicle + MCAO treated group). When the treatment was prolonged further to 7 days after MCAO, all groups including both positive control and the tomato pomace treated groups showed the significant increase in neurological score ( $p$-value $<0.01$, compared to vehicle + MCAO treated group). At 14 days after MCAO, the significant increased neurological scores were still observed (p-value $<0.01$ except Vitamin $\mathrm{C}$ treated group that showed $\mathrm{p}$-value $<0.05$, compared to vehicle + MCAO treated group).

We also assessed the infarct volume of various areas supplied by the middle cerebral artery. The results showed that rats subjected to Vitamin C treatment significantly decreased the infarct volume in hippocampus and striatum (p-value $<0.05$ and 0.01 respectively; compared to the vehicle treated group) as shown in Figure 2. The rats which received tomato pomace powder at dose of $2 \mathrm{mg} / \mathrm{kg}$ BW could significantly decrease the infarct volume in both hippocampus and cerebral cortex (pvalue $<0.05$ all; compared to the vehicle treated group). The increasing doses of tomato pomace powder to 10 and $50 \mathrm{mg} / \mathrm{kg}$ BW could produce significant reduction of brain infarct volume only in hippocampus ( $p$-value $<0.01$ all; compared to the vehicle + MCAO treated group).

\subsection{Effect of Tomato Pomace Powder on Oxidative Damage Status}

Since oxidative stress had been proposed to played the important role on the pathophysiology of brain damage induced by the occlusion of cerebral artery. It was found that although Vitamin $\mathrm{C}$ decreased the malondialdehyde (MDA) levels in hippocampus, striatum and cerebral cortex, no significant changes were observed. In addition, the significant reduction of MDA level in hippocampus was observed only in the rats subjected to high dose of tomato pomace powder ( $\mathrm{p}$-value $<0.05$; compared to the vehicle + MCAO treated group). The low dose treatment group significantly decreased the MDA level in both striatum and cerebral cortex (p-value $<0.05,0.01$ respectively; compared to the vehicle + MCAO treated group). It was revealed that the high dose treated group also produced the significant reduction of the mentioned parameter in both areas mentioned above (p-value $<0.05$ all; compared to the vehicle $+\mathrm{MCAO}$ treated group) as shown in Figure 3.

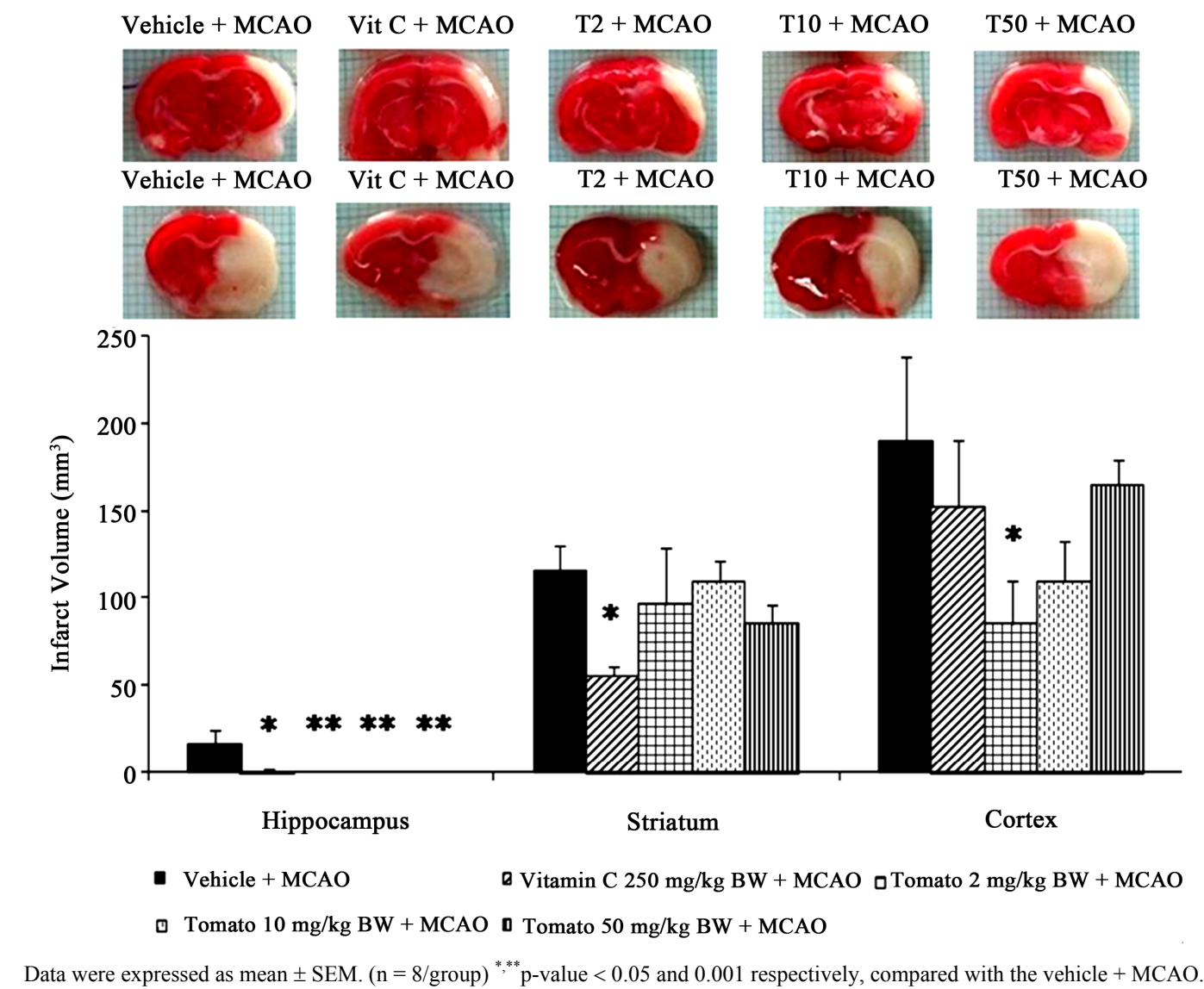

Figure 2. The effect of tomato pomace powder at doses of 2,10 and $50 \mathrm{mg} / \mathrm{kg} \mathrm{BW}$ on brain ischemic volume in hippocampus, striatum and cerebral cortex in animal model of focal stroke induced by the occlusion of right middle cerebral artery (MCAO). 
We also determined the alteration of superoxide dismutase (SOD) activity in various areas and the results were shown in Figure 4. The present results showed that rats which received Vitamin $\mathrm{C}$ treatment significantly increased the activity of SOD in both hippocampus and cerebral cortex ( $p$-value $<0.05$ all; compared to the vehicle + MCAO treated group). All doses of tomato pomace powder treated group could produce the significant elevation of SOD activity in hippocampus ( $p$-value $<0.05$ all; compared to the vehicle + MCAO treated group) while the significant elevation of this enzyme in striatum was observed in rats subjected to both medium and high doses of tomato pomace powder treatment ( $\mathrm{p}$-value < 0.001 all; compared to the vehicle + MCAO treated group). However, only the rats subjected to medium dose treatment significantly increased the SOD activity in cerebral cortex ( $\mathrm{p}$-value $<0.05$; compared to the vehicle + MCAO treated group).

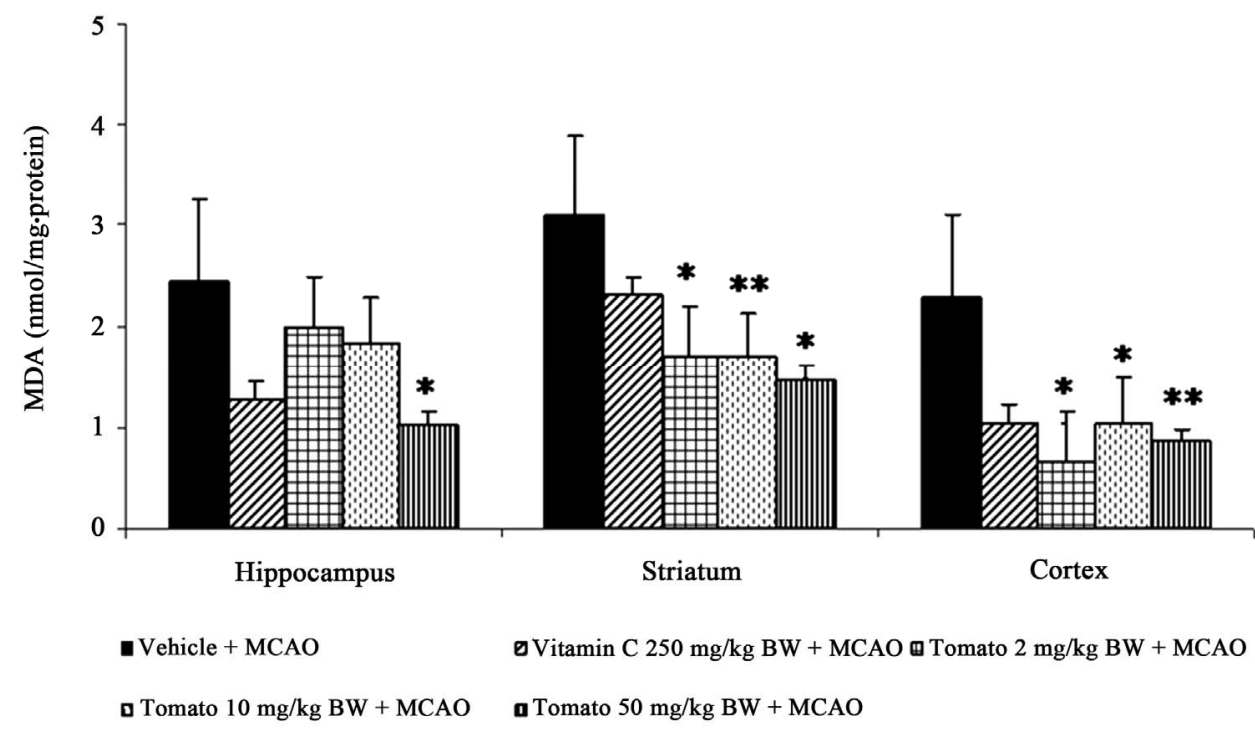

Data were expressed as mean \pm SEM. $\left(\mathrm{n}=8\right.$ /group) ${ }^{* * * *}$ p-value $<0.05$ and 0.01 respectively, compared with the vehicle + MCAO.

Figure 3. The effect of tomato pomace at doses of 2,10 and $50 \mathrm{mg} / \mathrm{kg} \mathrm{BW}$ on the level of malondialdehyde in hippocampus, striatum and cerebral cortex of animal model of focal stroke induced by the occlusion of right middle cerebral artery (MCAO).

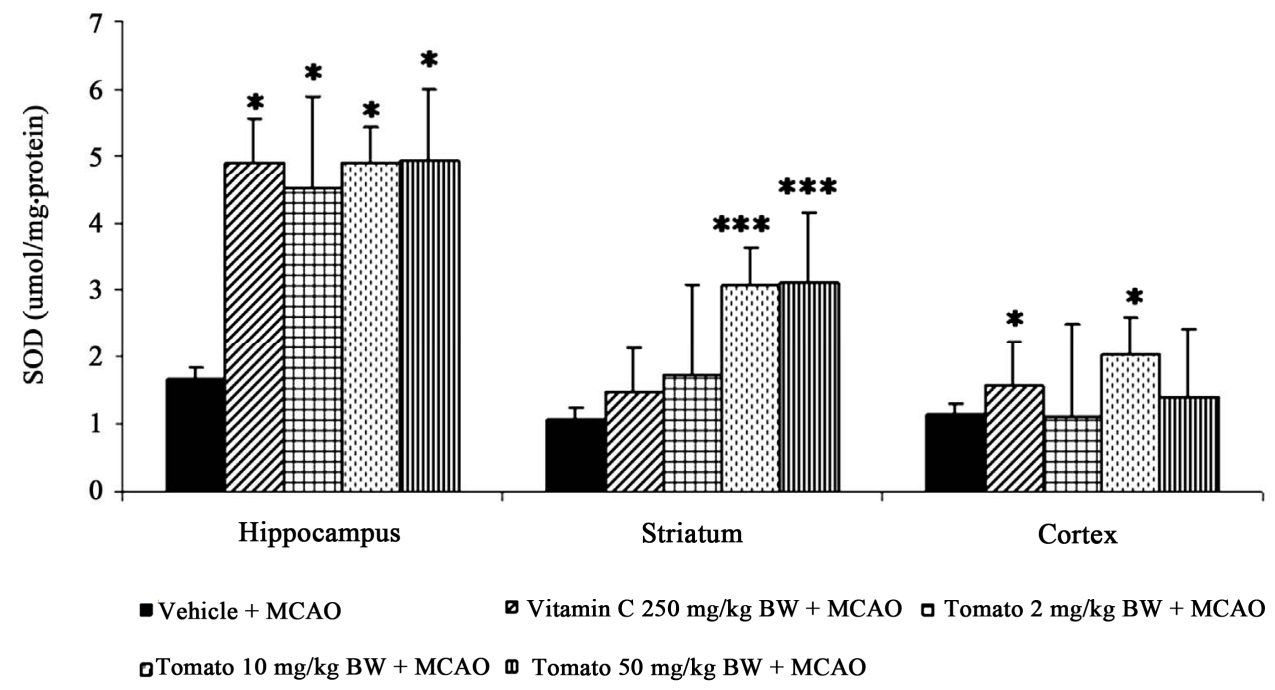

Data were expressed as mean \pm SEM. $\left(\mathrm{n}=8\right.$ /group) ${ }^{* * *}$ p-value $<0.05$ and 0.001 respectively, compared with the vehicle $+\mathrm{MCAO}$

Figure 4. The effect of tomato pomace powder at doses of 2,10 and $50 \mathrm{mg} / \mathrm{kg} \mathrm{BW}$ on the activity of superoxide dismutase (SOD) in hippocampus, striatum and cerebral cortex of animal model of focal stroke induced by the occlusion of right middle cerebral artery (MCAO). 
The alteration of catalase (CAT) and glutathione peroxidase (GSH-Px) activities in various areas mentioned earlier were also investigated and all results were shown in Figures 5 and 6. Our data clearly demonstrated that the positive control treated group significantly increased the catalase activity only in striatum ( $\mathrm{p}$-value $<0.05$; compared to the vehicle $+\mathrm{MCAO}$ treated group) while increased the activities of glutathione peroxidase (GSH$\mathrm{Px}$ ) in both hippocampus and cerebral cortex (p-value < 0.05 all; compared to the vehicle + MCAO treated group). The rats which received tomato pomace powder at dose of $50 \mathrm{mg} / \mathrm{kg}$ BW also enhanced GSH-Px activity in cerebral cortex ( $\mathrm{p}$-value $<0.05$; compared to the vehicle + MCAO treated group).

\section{Discussion}

In the current study, we have studied whether the tomato pomace powder can protect brain from cerebral ischemia induced by Rt. MCAO. It is well documented that permanent occlusion of middle cerebral artery (MCAO) gives rise to the neurological and histological abnormality. Our results indicated that the tomato pomace powder treatment offered the protection of various brain areas including hippocampus, striatum and cerebral cortex induced by MCAO. In accompany with the neuroprotection effect, the elevation of the activities of main scavenger enzymes, SOD and GSH-Px and the decreased MDA level were observed. It was previously demonstrated that

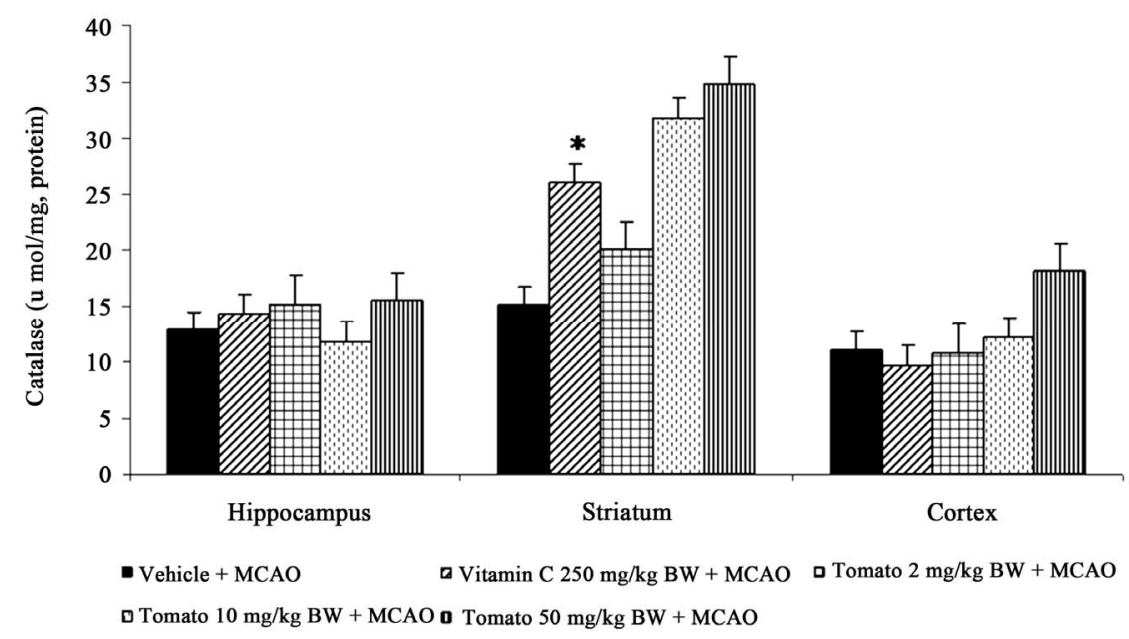

Data were expressed as mean \pm SEM. $\left(n=8\right.$ /group) ${ }^{*}$ p-value $<0.05$, compared with the vehicle + MCAO.

Figure 5. The effect of tomato pomace powder at doses of 2,10 and $50 \mathrm{mg} / \mathrm{kg} \mathrm{BW}$ on the activity of catalase (CAT) in hippocampus, striatum and cerebral cortex of animal model of focal stroke induced by the occlusion of right middle cerebral artery (MCAO).

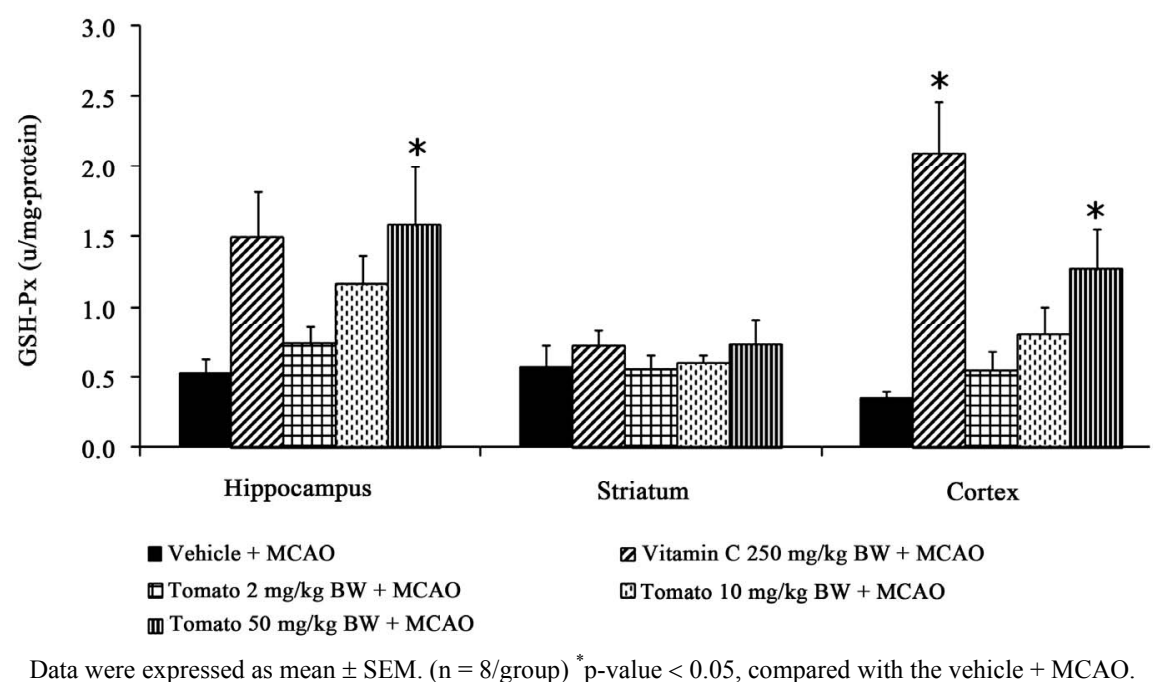

Figure 6. The effect of tomato pomace powder at doses of 2,10 and $50 \mathrm{mg} / \mathrm{kg} \mathrm{BW}$ on the activity of glutathione peroxidase (GSH-Px) in hippocampus, striatum and cerebral cortex of animal model of focal stroke induced by the occlusion of right middle cerebral artery (MCAO). 
the imbalance between the oxidative stress formation and the cell capability to manage oxidative stress. Recent study showed that the superoxide radical $\left(\mathrm{O}_{2}^{-}\right)$contributed the important role on the pathophysiology of cerebral ischemia [11]. Under normal circumstance, this radical was converted to $\mathrm{H}_{2} \mathrm{O}_{2}$ by SOD and then $\mathrm{H}_{2} \mathrm{O}_{2}$ was further detoxicated to $\mathrm{H}_{2} \mathrm{O}_{2}$ by catalase and GSH-Px. Therefore, tomato pomace at the dosage range used in this study might exert its neuroprotective effect partly by increasing the activities of both SOD and GSH-Px resulting in the decreased oxidative stress leading to the decreased brain infarction volume of the mentioned areas and finally enhanced the neurological score.

\section{Conclusion}

This study showed that the oral administration of tomato, a "Yin food" decreased the oxidative stress in rat brain by altering the levels of malondialdehyde but increased the activities of superoxide dismutase and glutathione peroxidase resulting in the neuroprotective effect of tomato against ischemic stroke, a Yin deficiency disorder. This implies that consumption of tomato may provide beneficial effect for those who are at risk for ischemic stroke. However, further researches are required to determine if these findings can be extended to prophylactic application tomato pomace powder for stroke.

\section{Acknowledgements}

This study was supported in part by the National Research Council of Thailand, the North Eastern Stroke Research Group and the Integrative Complimentary Alternative Medicine, Khon Kaen University, Khon Kaen, Thailand.

\section{REFERENCES}

[1] American Heart Association, "Heart Diseases and Stroke Statistics an Update," American Heart Association, Dallas, 2003.
[2] K. Baker, C. B. Marcus, K. Huffman, H. Kruk, B. Malfro and S. R. Doctrow, "Synthetic Combined Superoxide Dismutase/Catalase Mimetics Are Protective as a Delayed Treatment in a Rat Stroke Model: A Key Role for Reactive Oxygen Species in Ischemic Brain Injury," Journal of Pharmacological Experimental Therapeutic, Vol. 284, No. 1, 1998, pp. 215-221.

[3] A. R. Green and T. Ashwood, "Free Radical Trapping as a Therapeutic Approach to Neuroprotection in Stroke: Experimental and Clinical Studies with NXY-059 and Free Radical Scavengers," Current Drug Targets-CNS \& Neurological Disorders, Vol. 4, No. 2, 2005, pp. 109118. doi:10.2174/1568007053544156

[4] M. Barinaga, "Finding New Drugs to Treat Stroke," Science, Vol. 272, No. 5262, 1996, pp. 664-666.

[5] Y. Song, J. Pei, Z. D. liu, H. Y. Li, J. W. Guo and Y. Huang, "Dynamic Changes in Traditiona Chinese Medicine Syndromes in Patients with Ischemic Stroke Treated by Acupuncture," Journal of Chinese Integrative Medicine, Vol. 7, No. 4, 2009, pp. 334-341. doi:10.3736/jim20090407

[6] M. L. Nguyen and S. J. Schwartz, "Lycopene: Chemical and Biological Properties," Food Technology, Vol. 53, No. 2, 1999, pp. 38-45.

[7] J. Shi and M. Le Maguer, "Lycopene in Tomatoes: Chemical and Physical Properties Affected by Food Processing," Critical Review of Food Science and Nutrition, Vol. 40 No. 1, 2000, pp. 1-42. doi:10.1080/10408690091189275

[8] H. Wang, G. Cao and R. L. Prior, "Total Antioxidant Capacity of Fruits," Journal of Agricultural and Food Chemistry, Vol. 44, No. 3, 1996, pp. 701-705. doi:10.1021/jf950579y

[9] S. K. Clinton, "Lycopene: Chemistry, Biology, and Implications for Human Health and Disease," Nutrition Review, Vol. 56, No. 2, 1998, pp. 35-51. doi:10.1111/j.1753-4887.1998.tb01691.x

[10] F. Shahidi and P. K. J. P. D. Wanasundara, "Phenolic Antioxidants," Critical Reviews in Food Science and Nutrition, Vol. 32, 1992, pp. 67-103.

[11] D. S. Warner, H. Sheng and I. Batinić-Haberle, "Oxidants, Antioxidants and the Ischemic Brain," Journal of Experimental Biology, Vol. 207, 2004, pp. 3221-3231. 